\title{
Psoriasis in Patients of Color: Differences in Morphology, Clinical Presentation, and Treatment
}

\author{
Mathew N. Nicholas, MD; Airiss R. Chan, BSc; Morvarid Hessami-Booshehri, MD, FRCPC
}

\section{PRACTICE POINTS}

- There are key differences in psoriasis in patients with skin of color, including the morphology, clinical presentation, treatment, and psychosocial impact.

- Recognition and awareness of these differences may normalize the condition for patients, support them seeking medical attention sooner, and better inform them of all possible treatment options.

- Advocating further education on these differences in residency training and continuing medical education programs may help physicians make earlier diagnoses and personalize physician-patient conversations.

Psoriasis is a chronic inflammatory skin disease affecting $2 \%$ to $3 \%$ of individuals worldwide. However, the majority of clinical data are in white patients, with limited data in patients of color. We present 3 cases of psoriasis representative of patients presenting to an urban and racially diverse hospital-based dermatology clinic in Toronto, Ontario, Canada, to illustrate the differences in psoriasis in patients of color compared to white patients. We review the differences in the morphology, presentation, treatment, and psychosocial impact of psoriasis in this population. We also discuss the importance of early diagnosis, treatment considerations, and education in dermatology training programs regarding psoriasis in patients of color.

Cutis. 2020;106(suppl 2):7-10.

$\mathrm{P}$ soriasis is a chronic inflammatory skin disease that affects $2 \%$ to $3 \%$ of individuals worldwide. ${ }^{1}$ Despite extensive research, the majority of clinical data are in white patients with limited data in patients of color, yet a number of differences are known. The prevalence of psoriasis differs among racial and ethnic groups, with lower prevalence in racial minorities. ${ }^{2} \mathrm{~A}$ cross-sectional American study using data from 2009 through 2010 showed the prevalence for psoriasis was $3.6 \%$ in white patients, $1.9 \%$ in black patients, $1.6 \%$ in Hispanic patients, and $1.4 \%$ in other racial groups. ${ }^{3}$ Psoriasis presents differently in patients of color, both in morphology and severity. Cultural differences and stigma may contribute to the differences seen in severity but also to the psychological impact and treatment choices in patients of color compared to white patients. ${ }^{4}$ It has even been theorized that treatment efficacy could differ because of potential genetic differences. ${ }^{5}$ Psoriasis in patients of color is an emerging clinical issue that requires further attention so that dermatologists can learn about, diagnose, and treat them.

We report 3 cases of patients of color with psoriasis who presented to an urban and racially diverse dermatology clinic affiliated with Scarborough General Hospital in Toronto, Ontario, Canada. A retrospective chart review was performed on these high-yield representative cases to demonstrate differences in color and morphology, disease severity, and treatment in patients of various races seen at our clinic. After informed consent was obtained, photographs were taken of patient cutaneous findings to illustrate these differences. Discussion with these selected patients yielded supplementary qualitative data, highlighting individual perspectives of their disease.

\section{Case Series}

Patient 1-A 53-year-old black man from Grenada presented to our clinic with a history of psoriasis for a number of years that presented as violaceous plaques throughout large portions of the body (Figure 1). He previously had

From the University of Toronto, Ontario, Canada. Drs. Nicholas and Hessami-Booshehri are from the Division of Dermatology, Department of Medicine, and Ms. Chan is from the Faculty of Medicine. Dr. Nicholas also is from the Division of Dermatology, Women's College Hospital, Toronto. Dr. Hessami-Booshehri also is from Scarborough General Hospital, Ontario, Canada.

The authors report no conflict of interest.

The eTable is available in the Appendix online at www.mdedge.com/dermatology.

Correspondence: Mathew N. Nicholas, MD, Division of Dermatology, Women's College Hospital, 3rd Floor, 76 Grenville St, Toronto, ON M5S

1B2 Canada (mathew.nicholas@mail.utoronto.ca).

doi: $10.12788 /$ cutis.0038 
achieved inadequate results while using topical therapies, methotrexate, acitretin, apremilast, ustekinumab, ixekizumab, and guselkumab at adequate or even maximum doses. His disease affected $30 \%$ of the body surface area, with a psoriasis area and severity index score of 27 and a dermatology life quality index score of 23. The patient's life was quite affected by psoriasis, with emphasis on choice of clothing worn and effect on body image. He also discussed the stigma psoriasis may have in black patients, stating that he has been told multiple times that "black people do not get psoriasis."

Patient 2-A 27-year-old man from India presented with guttate psoriasis (Figure 2). He was treated with methotrexate 2 years prior and currently is on maintenance therapy with topical treatments alone. His main concerns pertained to the persistent dyschromia that occurred secondary to the psoriatic lesions. Through discussion, the patient stated that he "would do anything to get rid of it."

Patient 3-A 49-year-old man from the Philippines presented to our clinic with plaque psoriasis that predominantly affected the trunk and scalp (Figure 3). He had been treated with methotrexate and phototherapy with suboptimal efficacy and was planning for biologic therapy. Although he had active plaques on the trunk, the patient stated, "I am most bothered by my scalp," particularly referring to the itch and scale and their effects on hair and hairstyling.

\section{Comment}

Clinical differences in patients of color with psoriasis affect the management of the disease. Special consideration should be given to variances in morphology, presentation, treatment, and psychosocial factors in the management of psoriasis for these patient populations, as summarized in the eTable.

Morphology-At our clinic, patients of color have been found to have differences in morphology, including lesions that are more violaceous in color, as seen in patient 1; less noticeable inflammation; and more postinflammatory hypopigmentation and hyperpigmentation changes, as seen in patient 2 . These changes are supported by the literature and differ from typical psoriasis plaques, which are pink-red and have more overlying scale. The varied morphology also may affect the differential, and other mimickers may be considered, such as lichen planus, cutaneous lupus erythematosus, and sarcoidosis. ${ }^{2}$

Presentation-There are differences in presentation among patients of color, particularly in distribution, type of psoriasis, and severity. As seen in patient 3, Asian and black patients are more likely to present with scalp psoriasis. ${ }^{2,5}$ Hairstyling and hair care practices can differ considerably between racial groups. Given the differences in hairstyling, scalp psoriasis also may have a greater impact on patient quality of life (QOL).

Racial differences affect the type of psoriasis seen. Asian patients are more likely to present with pustular and erythrodermic psoriasis and less likely to present with inverse psoriasis compared to white patients. Hispanic patients are more likely to present with pustular psoriasis. ${ }^{11}$ Black patients have been reported to have lower frequencies of psoriatic arthritis compared to white patients. ${ }^{12}$ Recognition of these differences may help guide initial choice for therapeutics.

Notably, patients of color may present with much more severe psoriasis, particularly Asian and Hispanic patients. ${ }^{7}$ One retrospective study looking at patients with psoriasis treated with etanercept found that Asian patients were more likely to have greater baseline body surface area involvement. ${ }^{6}$ An American cross-sectional study reported higher psoriasis area and severity index scores in black patients compared to white patients, ${ }^{12}$
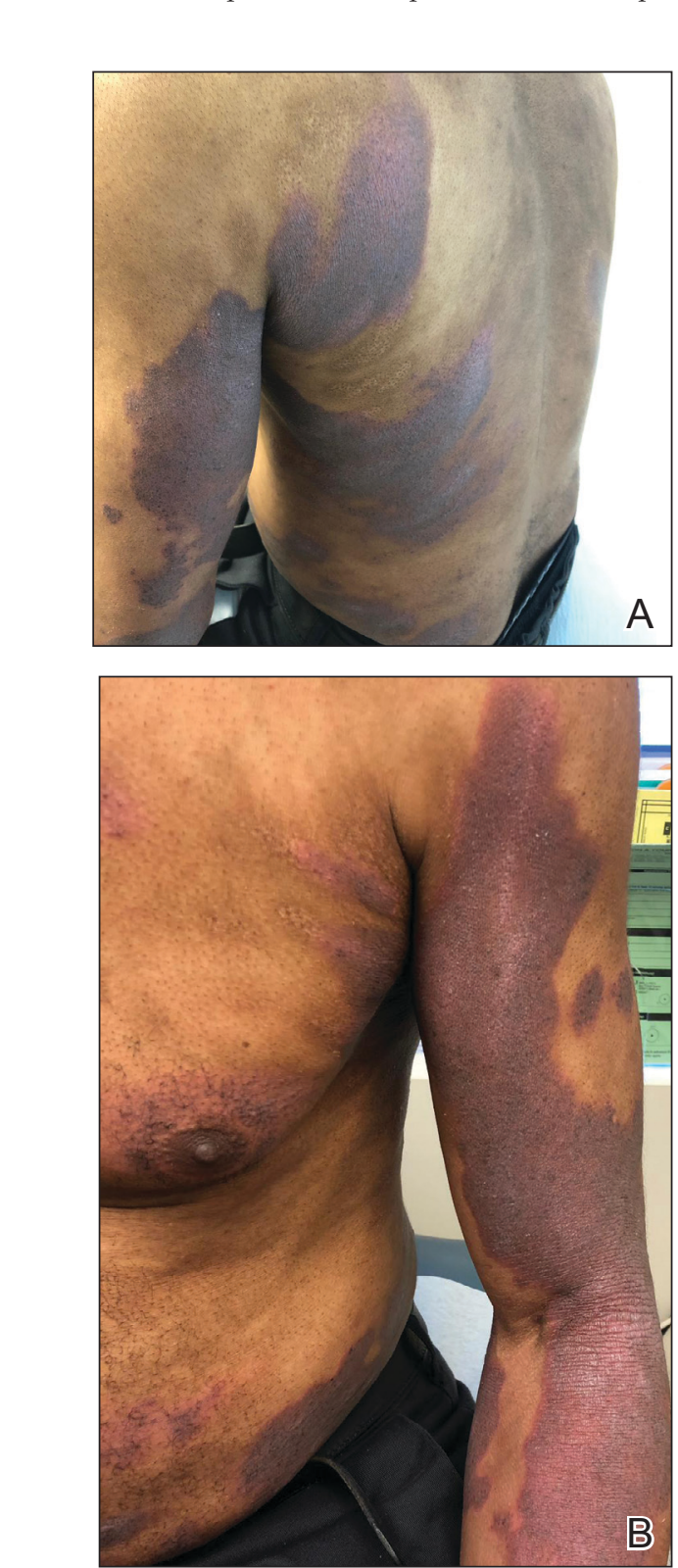

FIGURE 1. A and B, Violaceous plaques on the back and arm in a black patient with psoriasis. 
possibly because patients of color do not normalize the experience of having psoriasis and feel stigmatized, which can cause delays in seeking medical attention and worsen disease burden. For patient 1, the stigma of black patients having psoriasis affected his body image and may have led to a delay in seeking medical attention due to him not believing it was possible for people of his skin color to have psoriasis. Increased disease severity may contribute to treatment resistance or numerous trials of topicals or biologics before the disease improves. Patient education in the community as well as patient support groups are paramount, and increased awareness of psoriasis can help improve disease management.

Treatment-Topical therapies are the first-line treatment of psoriasis. Although there is no evidence showing


FIGURE 2. A and B, Postinflammatory hyperpigmentation from guttate psoriasis in a patient from India. differences in topical treatment efficacy, patient preference for different topical treatments may vary based on race. For example, patients with Afro-textured hair may prefer foams and lotions and would avoid shampoo therapies, as frequent hair washing may not be feasible with certain hairstyles and may cause hair breakage or dryness. ${ }^{2}$

UV therapy can be an effective treatment modality for patients with psoriasis. The strength of therapy tends to be dictated by the Fitzpatrick skin phototype rather than race. Darker-skinned individuals may have an increased risk for hyperpigmentation, so caution should be taken to prevent burning during therapy. Suberythemogenic dosing-70\% of minimal erythema dose-of narrowband UVB treatments has shown the same efficacy as using minimal erythema dose in patients with darker skin types in addition to fair-skinned patients. ${ }^{8}$

Although we found poor efficacy of systemic treatments in patient 1, to our knowledge, studies examining the efficacy of systemic therapeutic options have not shown differences in patients of color. ${ }^{6,13}$ Studies show similar efficacy in treatments among races, particularly biologic therapies. ${ }^{5}$ However, patients with skin of color historically have been underrepresented in clinical trials, ${ }^{9}$
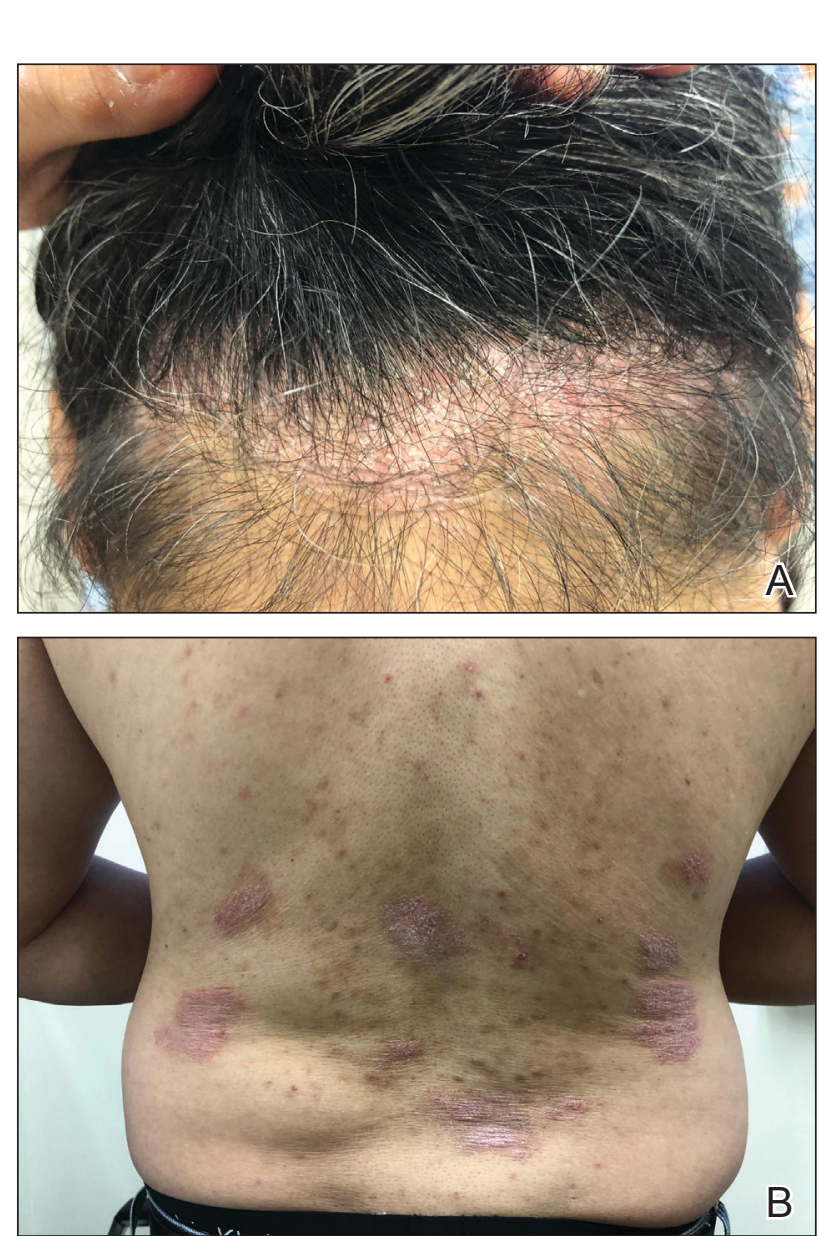

FIGURE 3. A and B, Plaque psoriasis on the scalp and back, respectively, in a patient from the Philippines. 
which may contribute to these patients, particularly black patients, being less familiar with biologics as a treatment option for psoriasis, as reported by Takeshita et al. ${ }^{10}$ Therefore, patient-centered discussions regarding treatment choices are important to ensure patients understand all options available to manage their disease.

Psychosocial Impact-Because of its chronic remitting course, psoriasis has a notable psychosocial impact on the lives of all patients, though the literature suggests there may be more of an impact on QOL in patients of color. Higher baseline dermatology life quality index scores have been reported in patients of color compared to white patients. ${ }^{6}$ Kerr et $\mathrm{al}^{12}$ reported significantly greater psoriasis area and severity index scores $(P=.06)$ and greater psychological impact in black patients compared to white patients. Stress also was more likely to be reported as a trigger for psoriasis in patients of Hispanic background compared to white patients. ${ }^{14}$ Many patients report body image issues with large physical lesions; however, the difference may lie in personal and cultural views about psoriasis, as one of our patients stated, "black people do not get psoriasis." In addition to the cosmetic challenges that patients face with active lesions, postinflammatory pigmentary changes can be equally as burdensome to patients, as one of our patients stated he "would do anything to get rid of it." Increased rates of depression and anxiety in patients of color can worsen their outlook on the condition. ${ }^{15,16}$ The increased stigma and burden of psoriasis in patients of color calls for clinicians to counsel and address psoriasis in a holistic way and refer patients to psoriasis support groups when appropriate. Although the burden of psoriasis is clear, more studies can be carried out to investigate the impact on QOL in different ethnic populations.

Dermatology Education-Although differences have been found in patients of color with psoriasis, dissemination of this knowledge continues to be a challenge. In dermatology residency programs, the majority of teaching is provided with examples of skin diseases in white patients, which can complicate pattern recognition and diagnostic ability for trainees. Although dermatologists recognize that ethnic skin has unique dermatologic considerations, there is a persistent need for increasing skin of color education within dermatology residency programs. ${ }^{17,18}$ Implementing more educational programs on skin of color has been proposed, and these programs will continue to be in demand as our population increasingly diversifies. ${ }^{19}$

\section{Conclusion}

Psoriasis in patients of color carries unique challenges when compared to psoriasis in white patients. Differences in morphology and presentation can make the disease difficult to accurately diagnose. These differences in addition to cultural differences may contribute to a greater impact on QOL and psychological health. Although treatment preferences and recognition may differ, treatment efficacy has so far been similar, albeit with a low proportion of patients with skin of color included in clinical trials.

Further focus should now lie within knowledge translation of these differences, which would normalize the condition for patients, support them seeking medical attention sooner, and inform them of all treatment options possible. For clinicians, more attention on the differences would help make earlier diagnoses, personalize physician-patient conversations, and advocate for further education on this issue in residency training programs.

\section{REFERENCES}

1. National Psoriasis Foundation. Statistics. https://www.psoriasis.org /content/statistics. Accessed July 14, 2020.

2. Alexis AF, Blackcloud P. Psoriasis in skin of color: epidemiology, genetics, clinical presentation, and treatment nuances. J Clin Aesthet Dermatol. 2014;7:16-24.

3. Rachakonda TD, Schupp CW, Armstrong AW. Psoriasis prevalence among adults in the United States. J Am Acad Dermatol. 2014;70:512-516.

4. Goff KL, Karimkhani C, Boyers LN, et al. The global burden of psoriatic skin disease. BrJ Dermatol. 2015;172:1665-1668.

5. Kaufman BP, Alexis AF. Psoriasis in skin of color: insights into the epidemiology, clinical presentation, genetics, quality-of-life impact, and treatment of psoriasis in non-white racial/ethnic groups. Am J Clin Dermatol. 2018;19:405-423.

6. Shah SK, Arthur A, Yang YC, et al. A retrospective study to investigate racial and ethnic variations in the treatment of psoriasis with etanercept. J Drugs Dermatol. 2011;10:866-872.

7. Abrouk M, Lee K, Brodsky M, et al. Ethnicity affects the presenting severity of psoriasis. J Am Acad Dermatol. 2017;77:180-182.

8. Youssef RM, Mahgoub D, Mashaly HM, et al. Different narrowband UVB dosage regimens in dark skinned psoriatics: a preliminary study. Photodermatol Photoimmunol Photomed. 2008;24:256-259.

9. Charrow A, Xia F Di, Joyce C, et al. Diversity in dermatology clinical trials: a systematic review. JAMA Dermatol. 2017;153:193-198.

10. Takeshita J, Eriksen WT, Raziano VT, et al. Racial differences in perceptions of psoriasis therapies: implications for racial disparities in psoriasis treatment. J Invest Dermatol. 2019; 139:1672-1679.

11. Yan D, Afifi L, Jeon C, et al. A cross-sectional study of the distribution of psoriasis subtypes in different ethno-racial groups. Dermatol Online J. 2018;24. pii:13030/qt5z21q4k2.

12. Kerr GS, Qaiyumi S, Richards J, et al. Psoriasis and psoriatic arthritis in African-American patients-the need to measure disease burden. Clin Rheumatol. 2015;34:1753-1759.

13. Edson-Heredia E, Sterling KL, Alatorre CI, et al. Heterogeneity of response to biologic treatment: perspective for psoriasis. I Invest Dermatol. 2014;134:18-23.

14. Yan D, Afifi L, Jeon C, et al. A cross-sectional study of psoriasis triggers among different ethno-racial groups. J Am Acad Dermatol. 2017;77:756-758.

15. Bailey RK, Mokonogho J, Kumar A. Racial and ethnic differences in depression: current perspectives. Neuropsychiatr Dis Treat. 2019;15:603-609.

16. Jackson C, Maibach H. Ethnic and socioeconomic disparities in dermatology. J Dermatolog Treat. 2016;27:290-291.

17. Salam A, Dadzie OE. Dermatology training in the U.K.: does it reflect the changing demographics of our population? Br J Dermatol. 2013;169:1360-1362.

18. Nijhawan RI, Jacob SE, Woolery-Lloyd H. Skin of color education in dermatology residency programs: does residency training reflect the changing demographics of the United States? J Am Acad Dermatol. 2008;59:615-618.

19. Ogunyemi B, Miller-Monthrope Y. The state of ethnic dermatology in Canada. J Cutan Med Surg. 2017;21:464-466. 


\section{APPENDIX}

eTABLE. Notable Differences in Psoriasis in Patients of Color

\begin{tabular}{|c|c|}
\hline Type of Difference & Characteristic \\
\hline \multirow[t]{3}{*}{ Morphology } & More violaceous in color ${ }^{2, a}$ \\
\hline & Less noticeable inflammation ${ }^{2, a}$ \\
\hline & $\begin{array}{l}\text { More postinflammatory } \\
\text { hypopigmentation and } \\
\text { hyperpigmentation } 2, a\end{array}$ \\
\hline \multirow[t]{4}{*}{ Presentation } & $\begin{array}{l}\text { More scalp psoriasis in Asian and } \\
\text { black patients }{ }^{5}\end{array}$ \\
\hline & $\begin{array}{l}\text { Potentially greater impact on } \mathrm{QOL} \\
\text { with scalp psoriasis }\end{array}$ \\
\hline & $\begin{array}{l}\text { Greater BSA involvement in Asian } \\
\text { patients }^{6}\end{array}$ \\
\hline & $\begin{array}{l}\text { More severe disease at } \\
\text { presentation } 7, a\end{array}$ \\
\hline \multirow[t]{5}{*}{ Treatment } & $\begin{array}{l}\text { Preference for foams and lotions } \\
\text { for scalp } p^{2, a}\end{array}$ \\
\hline & $\begin{array}{l}\text { UV consideration for prevent } \\
\text { hyperpigmentation }^{8}\end{array}$ \\
\hline & $\begin{array}{l}\text { No known differences in efficacy } \\
\text { of systemic treatments }{ }^{5}\end{array}$ \\
\hline & $\begin{array}{l}\text { Patients with skin of color } \\
\text { underrepresented in clinical trials }\end{array}$ \\
\hline & $\begin{array}{l}\text { Reduced patient recognition of } \\
\text { certain treatments }{ }^{10}\end{array}$ \\
\hline Psychosocial imp & Higher DLQI at baseline ${ }^{6, a}$ \\
\hline
\end{tabular}

Abbreviations: QOL, quality of life; BSA, body surface area; DLQI, dermatology life quality index.

aNoted at Scarborough General Hospital, Ontario, Canada. 